\title{
Letter to the Editor: Could Immunogenicity of Kaposi Sarcoma Be More Linked to Viral Antigens Than to the Tumor Mutational Burden?
}

Re: James Saller, Christine M. Walko, Sherri Z. Millis, et al. Response to Checkpoint Inhibitor Therapy in Advanced Classic Kaposi Sarcoma: A Case Report and Immunogenomic Study. J Natl Compr Canc Netw 2018;16(7):797-800.

We read with great interest the article by Saller et al, ${ }^{1}$ which extends the observations of our previous report of 2 cases of classic Kaposi sarcoma (KS) successfully treated with PD-1 blockade. ${ }^{2}$

Although both of our patients similarly experienced durable partial responses to PD-1 inhibition, it is of paramount importance to keep in mind that up to $15 \%$ of patients may experience severe immune-related adverse events (irAEs), most often requiring the administration of high doses of steroids and/or immunosuppressive treatments. Although these treatments are essential for the management of irAEs, they are also associated with KS promotion, including classic KS. ${ }^{3}$ Therefore, the benefits of immune checkpoint inhibition (ICI) may be overshadowed. Indeed, one of our patients developed severe immune-related myocarditis, resulting in acute pulmonary edema and cardiogenic shock, with a left ventricular ejection fraction reduction to $25 \%$, requiring several days of noninvasive ventilation and inotropic support. The myocarditis was successfully treated with high doses of intravenous methylprednisolone and immunoglobulin therapy, followed by 6 weeks of systemic corticosteroids. However, KS subsequently relapsed within 5 months with major bone involvement, leading to the reinstitution of chemotherapy.

We agree that a high tumor mutational burden (TMB) is generally associated with an increased tumor response to ICI. However, other markers have recently been unveiled, such as PD-L1 amplification $^{4}$ and, most importantly, immunogenicity of virus-associated antigens in virus-associated tumors. It is worth emphasizing that in Merkel cell polyomavirus (MCPyV)-associated Merkel cell carcinoma (MCC), which represents up to $80 \%$ of cases in the United States and Europe, TMB has been shown to be low (12.5 mutations per exome), whereas the benefit from $\mathrm{ICI}$ is at least as good as in MCPyV-negative, UV-induced MCC, which carries a high TMB (1,121 mutations per exome). ${ }^{5}$ It is now known that all KS are associated with HHV-8 infection. Cytogenetic and comparative genomic hybridization studies have revealed few abnormalities in early KS, such as clonal loss of chromosome $\mathrm{Y}$, whereas additional chromosomal aberrations can be detected in more advanced stages of the disease. ${ }^{6}$ Therefore, we hypothesize that the strong response to PD-1 inhibition observed in KS may probably be driven more by immunogenic viral antigens than by the TMB.

Further studies are warranted to confirm these results and decipher the importance of immunogenic viral antigens and/or TMB. The hope is that results of an ongoing French multicentric study will provide further insight into the benefit of PD-1 inhibition in classic/ endemic KS (ClinicalTrials.gov identifier: NCT03469804).

Julie Delyon, $\mathrm{MD}, \mathrm{PhD}$ Hôpital Saint-Louis,

Paris Diderot University, Paris, France Email: julie.delyon@aphp.fr

Dimitri Arangalage, $\mathrm{MD}, \mathrm{PhD}$ Hôpital Bichat,

Paris Diderot University, Paris, France

Barouyr Baroudjian, MD Hôpital Saint-Louis, Paris, France
Call for Correspondence JNCCN is committed to providing a forum to enhance collaboration between academic medicine and the community physician. We welcome comments about the NCCN Clinical Practice Guidelines in Oncology (NCCN Guidelines), articles published in the journal, or any other topic relating to cancer prevention, detection, treatment, supportive care, or survivorship.

Please send correspondence to JNCCN.edmgr.com or to JNCCN@nccn.org.

Letters should be no more than 400 words, with no more than 5 references if included. Please include the full names, degrees, and affiliations of all letter authors and a phone number or e-mail address for contact.

Letters are considered for publication as space allows. NCCN reserves the right not to publish correspondence for any reason it deems appropriate. All letters are subject to editing and/or abridgment. 
Celeste Lebbe, MD, PhD

Hôpital Saint-Louis, Paris Diderot University

Paris, France

doi: $10.6004 /$ jnccn.2018.7088

\section{References}

1. Saller J, Walko CM, Millis SZ, et al. Response to checkpoint inhibitor therapy in advanced classic Kaposi sarcoma: a case report and immunogenomic study. J Natl Compr Canc Netw 2018;16:797-800.

2. Delyon J, Bizot A, Battistella M, et al. PD-1 blockade with nivolumab in endemic Kaposi sarcoma. Ann Oncol 2018;29:1067-1069.

3. Anderson LA, Lauria C, Romano $\mathrm{N}$, et al. Risk factors for classical Kaposi sarcoma in a population-based case-control study in Sicily. Cancer Epidemiol Biomarkers Prev 2008;17:3435-3443.

4. Goodman AM, Piccioni D, Kato S, et al. Prevalence of PDL1 amplification and preliminary response to immune checkpoint blockade in solid tumors. JAMA Oncol 2018;4:1237-1244.

5. Nghiem PT, Bhatia S, Lipson EJ, et al. PD-1 blockade with pembrolizumab in advanced Merkel-cell carcinoma. N Engl J Med 2016;374:2542-2552.

6. Sun Z, Xiao B, Jha HC, et al. Kaposi's sarcoma-associated herpesvirus-encoded LANA can induce chromosomal instability through targeted degradation of the mitotic checkpoint kinase bub1. J Virol 2014;88:7367-7378.

\section{Authors' Reply to Letter to the Editor: Could Immunogenicity of Kaposi Sarcoma Be More Linked to Viral Antigens Than to the Tumor Mutational Burden?}

\author{
Authors' Reply to Julie Delyon, Dimitri Arangalage, \\ Barouyr Baroudjian, Celeste Lebbe's Letter to the \\ Editor re: James Saller, Christine M. Walko, Sherri Z. \\ Millis, et al. Response to Checkpoint Inhibitor Therapy \\ in Advanced Classic Kaposi Sarcoma: A Case Report \\ and Immunogenomic Study. J Natl Compr Canc Netw \\ 2018;16(7):797-800.
}

We thank Delyon et al for their interest in our recent report and immunogenomics study in classic Kaposi sarcoma. ${ }^{1}$ We agree that our case, along with their contemporaneously reported 2-patient series, ${ }^{2}$ lend strong anecdotal evidence to support further evaluation of immune checkpoint inhibition (ICI) in this rare disease. We also agree that responsiveness to ICI is almost certainly not exclusively determined by somatic tumor mutational burden, but likely is linked to a multitude of factors.

We caution, however, against speculating that viral antigens are the likely driver of ICI response in this disease. Although response rates to ICIs in several viral-driven malignancies have been encouraging, it is not clear that viral antigens are the primary driver of these responses. A recent report in human papillomavirus (HPV)-related cervical cancer suggests the opposite. ${ }^{3}$ In this report, the antitumor T-cell responses in patients who experienced complete response to adoptive cell therapy were found to be predominantly directed against nonviral antigens rather than against virally associated antigens. This result suggests that success of immunotherapy in virus-driven malignancies might be related to other viral factors (eg, microenvironmental changes, induction of immune checkpoint expression), rather than viral antigens themselves being the driving source of immunoreactivity. In this paradigm, tumor antigens such as somatic neoantigens or cancer testes antigens would therefore be required for ICI response.

We share in the enthusiasm for further studies to evaluate the determinants of ICI response in Kaposi sarcoma and establish the rate of efficacy of ICIs in this disease.

$$
\begin{array}{r}
\text { Andrew S. Brohl, MD } \\
\text { Moffitt Cancer Center and Research Institute } \\
\text { Tampa, FL } \\
\text { Email: andrew.broh@moffitt.org } \\
\text { James Saller, MD } \\
\text { Moffitt Cancer Center and Research Institute } \\
\text { Tampa, FL } \\
\text { doi: 10.6004/jnccn.2018.7087 }
\end{array}
$$

\section{References}

1. Saller J, Walko CM, Millis SZ, et al. Response to checkpoint inhibitor therapy in advanced classic Kaposi sarcoma: a case report and immunogenomic study. J Natl Compr Canc Netw 2018;16:797-800.

2. Delyon J, Bizot A, Battistella M, et al. PD-1 blockade with nivolumab in endemic Kaposi sarcoma. Ann Oncol 2018;29:1067-1069.

3. Stevanovic S, Pasetto A, Helman SR, et al. Landscape of immunogenic tumor antigens in successful immunotherapy of virally induced epithelial cancer. Science 2017;356:200-205. 